\title{
CHOREA AS A CLINICAL FEATURE OF SYSTEMIC LUPUS ERYTHEMATOSUS - SYSTEMATIC REVIEW
}

Lucas de Moura Brito ${ }^{1}$, Rafael Cartaxo Nascimento ${ }^{1}$, Evânia Claudino Queiroga de Figueiredo ${ }^{1, \star}$, Marcus Ivanovith Fernandes ${ }^{1}$, Bárbara Letícia de Moura Pinheiro ${ }^{1}$, Priscylla Emylly Lacerda de Sousa1, Antônio da Silva Ramos Segundo Neto ${ }^{1}$, Thamiris Miranda Granja ${ }^{1}$

1.Universidade Federal de Campina Grande, Campina Grande (PB), Brazil.

*Corresponding author: evaniaclaudinofig@gmail.com

\section{BACKGROUND}

Systemic lupus erythematosus (SLE) is a pathology with potentially serious systemic involvement and often difficult to diagnose. Sometimes atypical manifestations occur, such as chorea, which is associated with the presence of antiphospholipid antibodies in the context of SLE. This clinical manifestation is more common in other diagnoses, such as rheumatic fever. In this context, it is necessary to clarify the relationship of chorea in the clinical course of SLE.

\section{METHODS}

Identify the epidemiological, clinical and laboratory characteristics of patients with SLE and chorea, through a systematic literature review using reports and case series from the Medical Literature and Retrieval System Online (MEDLINE) and Literatura Latino-Americana e do Caribe em Ciências da Saúde (LILACS) databases.

\section{RESULTS}

Forty-nine case reports were analyzed, 44 of which were selected for data analysis, after 5 exclusions due to the absence of numerous variables. Most patients were female 30/44 (68.2\%). In half of the case reports, chorea manifested itself in the context of pediatric SLE. In 30/44 (68.2\%) of cases, chorea appeared before the diagnosis of SLE. The most considered differential diagnosis was rheumatic fever, with a prevalence of $86.6 \%$ in the reports that described the possible differential diagnoses. There was the presence of 31/44 (70.5\%) positive anti-dsDNA antibody and 24/44 (54.5\%) with hypocomplementemia, the two most prevalent findings. In 29/33 (87.8\%) of patients in whom antiphospholipid antibodies (aPL) were investigated, at least one of them was positive. Magnetic resonance imaging (MRI) was unchanged in 10/22 (45.4\%) of patients who underwent this examination.

\section{CONCLUSION}

Our study suggested a higher prevalence of SLE associated with chorea in young patients, during disease activity. This was evidenced by both clinical and laboratory tests. The classic relationship with aPL was noted in the reports. In addition, MRI does not show changes in most cases, but it can be used to search for differential diagnoses.

\section{KEYWORDS}

Systemic lupus erythematosus, Antiphospholipid antibody, Chorea, Movement disorders. 